\title{
Diacronie
}

Studi di Storia Contemporanea

$N^{\circ} 9,1 \mid 2012$

Quando la classe operaia andava in paradiso

\section{Eretici e libertari}

Il movimento anarchico in Italia (1945-1973)

\section{Giorgio Sacchetti}

\section{(2) OpenEdition \\ Journals}

\section{Edizione digitale}

URL: http://journals.openedition.org/diacronie/2991

DOI: 10.4000/diacronie. 2991

ISSN: 2038-0925

Editore

Association culturelle Diacronie

Notizia bibliografica digitale

Giorgio Sacchetti, «Eretici e libertari », Diacronie [Online], № 9, 1 | 2012, documento 8, Messo online il 29 janvier 2012, consultato il 21 avril 2019. URL : http://journals.openedition.org/diacronie/2991 ;

DOI : 10.4000/diacronie.2991 


\title{
Diacronie
}

N. 9 | 1|2012 Quando la classe operaia andava in paradiso

8/

\section{Eretici e libertari}

\section{Il movimento anarchico in Italia (1945-1973)}

\author{
Giorgio SACCHETTI*
}

Heri dicebamus. Dopo la Resistenza gli anarchici riprendono il filo del loro discorso rivoluzionario nel nuovo contesto politico e sociale italiano. Decimati nella lunga lotta antifascista, sconfitti nella guerra di Spagna, spettatori inermi della bolscevizzazione del movimento operaio i vecchi militanti si apprestano al duro passaggio dal protagonismo di massa primo novecentesco alla testimonianza. Così il movimento si rigenera in una sorta di "neo-anarchismo" attraverso contaminazioni culturali con la sinistra eretica degli anni Cinquanta, con i movimenti libertari del decennio successivo. Quattro le questioni salienti che connotano le vicende dell'anarchismo nell'Italia repubblicana: la FAI nuovo soggetto politico; l'anarcosindacalismo; la dimensione internazionale; il Sessantotto.

\section{La federazione anarchica italiana}

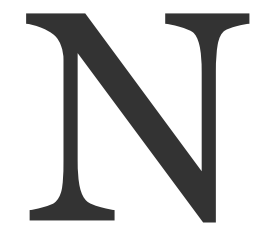

el dopoguerra l'anarchismo perde la sua base di classe in coincidenza delle profonde trasformazioni del paese. La militanza partigiana come lotta di liberazione nazionale contro l'occupante tedesco, il richiamo al risorgimento, il mito sovietico, sono i contenuti d'impatto nella transizione alla democrazia. L'antifascismo, convertito in sistema di governo, è ora elemento di ricomposizione tra politico e statale. Il PCI, complici sviluppo dei partiti di massa e strategia togliattiana, raccoglie a sinistra l'eredità del sovversivismo. Il restante ridimensionamento si compirà con la guerra fredda. Anche nelle zone a consolidata 
tradizione libertaria vi sono scollamenti nella "base"; specie al referendum del $1946^{1} \mathrm{e}$ alle elezioni del 1948, appuntamenti del "non ritorno". Integratosi il movimento operaio nello Stato, inizia la normalizzazione. La fase delle opportunità antifasciste radicali si rivela effimera; si apre quella invece dell'abbandono delle grandi speranze. Così la nostalgia politica è base troppo fragile per creatività e immaginazione sociale (almeno fino al risveglio degli ancora lontani anni Sessanta).

Varie le ricomposizioni organizzative a latere della Federazione Anarchica Italiana (FAI) fondata nel 1945: i Gruppi Anarchici di Azione Proletaria (GAAP) sorti nel 1951; la scissione dalla FAI, nel 1965, dei Gruppi di Iniziativa Anarchica (GIA) e la nascita dei Gruppi Anarchici Federati (GAF). A fianco di questo percorso si colloca la straordinaria esperienza culturale della rivista «Volontà» diretta, fino al 1962, da Giovanna Caleffi, vedova di Camillo Berneri. Punto di raccordo europeo e di fecondo dialogo fra libertari e sinistra eretica. Nelle sue pagine trova spazio l'Italia "minoritaria" degli anni Cinquanta (Gaetano Salvemini, Lamberto Borghi, Adriano Olivetti, Angelo Tasca, Aldo Capitini, Ignazio Silone, Ernesto Rossi, Margherita Zoebeli, don Lorenzo Milani...).

A fine ' 44 quando, a Roma, vede di nuovo la luce «Umanità Nova», numero unico della Federazione Comunista Libertaria Italiana (FCL), poi settimanale. Dal 1945 la FCL già dispone di un proprio giornale nazionale, in parte finanziato da anarchici italoamericani. Alla vigilia della costituzione della FAI ferve il dibattito sul programma approvato dai confinati anarchici a Ventotene. Si riafferma l'autonomia da partiti e CLN, pur nella ricerca di accordi con la sinistra rivoluzionaria anticapitalista.

$\mathrm{Al}$ congresso di Carrara (14-20 settembre 1945) partecipano 276 delegati. Neutralità dell'Italia e rifiuto delle spese militari; parità per le donne; azione diretta contro i proprietari terrieri; sistemi produttivi a gestione collettiva; finanziamenti all'edilizia gestiti localmente; scuola libera e gratuita sono gli obiettivi dichiarati. Si delibera la costituzione della FAI, organizzazione assembleare, federalista, autogestita e senza funzionari stipendiati; fra gli organi: Consiglio nazionale, comitato sindacale ("Gruppi di Difesa Sindacalista" nella CGIL). Il clima di grandi speranze e la fede nei "sacri" principi non bastano però a celare i primi dissidi² .

\footnotetext{
${ }^{1}$ Sul referendum prevale la posizione astensionista rispetto ai sostenitori del voto per la repubblica. Cfr. BORGHI, Armando, Conferma anarchica. (Due anni in Italia), Forlì, L'Aurora, 1949, p. 91; «Il Libertario», Milano, a. 1946, pass.

2 Cfr. ROSSI, Italino, La ripresa del movimento anarchico italiano e la propaganda orale dal 1943 al 1950, Pistoia, RL, 1981; SACCHETTI, Giorgio, Sovversivi agli atti. Gli anarchici nelle carte del Ministero dellinterno. Schedatura e controllo poliziesco nell'Italia del Novecento, Ragusa, La Fiaccola, 2002; ILARI, Massimiliano, Parole in libertà. Il giornale anarchico Umanità Nova (1944-1953), Milano, Zero in Condotta, 2009.
} 
Al II congresso della FAI (Bologna, marzo 1947) si palesa la svolta "antiorganizzatrice” perorata dagli italo-americani de «L'Adunata dei Refrattari». Il Consiglio nazionale è sostituito con la Commissione di Corrispondenza (C.d.C.), detta la "buca delle lettere".

Esaurita la spinta propulsiva resistenziale, la FAI deve fare i conti con la propria storia, pagando con la diaspora dei comunisti-libertari. La guerra fredda confina il "terzaforzismo" nella testimonianza, fra stalinizzazione del movimento operaio e persecuzioni poliziesche.

Al III congresso della FAI (Livorno, aprile 1949) si formalizza una corrente per un "movimento orientato e federato"; situazione foriera di nuovi sviluppi, conferma dell'incompatibilità tra "classisti" e "aclassisti", fra rivoluzione proletaria e lotta umana. I contrasti fra gli "anti-organizzatori”, appoggiati da «Volontà», e la corrente che fa capo a Pier Carlo Masini costringono quest'ultimo a dimettersi da «Umanità Nova». Sul nuovo periodico «L'Impulso» gli “orientatori” attaccano la FAI «coacervo eterogeneo, tenuto insieme da vecchie e scarse riserve di sentimentalismo»3.

Al IV congresso della FAI (Ancona, dicembre 1950) si dibatte sull'intervento sindacale. Prevale la linea di Armando Borghi, contraria sia alla riattivazione dell'Unione Sindacale Italiana (USI), sia all'entrismo nella CGIL. A seguito del deliberato che «conferma il concetto tradizionale di movimento anarchico aperto non esclusivamente politico», si verifica la fuoriuscita degli “orientatori”. Questi si costituiscono (1951) in GAAP. Contro l'immobilismo degli anarchici italiani, il documento programmatico della nuova organizzazione, rilancia l'iniziativa della Federazione Francese per un Terzo Fronte anti-imperialista 4 .

Dopo la scissione la consistenza della FAI è così raffigurata da Fedeli5:

[...] «Umanità Nova» stampa sulle quindicimila copie e «Il Libertario» attorno alle dodicimila, il «Seme Anarchico» di propaganda semplice ottomila e la rivista «Volontà» attorno alle duemila. La FAI si compone di almeno trecento gruppi sparsi in tutta Italia e riuniti in Federazioni locali e in Federazioni regionali, delle

\footnotetext{
${ }_{3}$ L'Impulso, n. 9-10/1950.

4 Cfr. GRUPPI ANARCHICI D'AZIONE PROLETARIA, Sulla liquidazione dello Stato come apparato di classe (Tesi programmatiche approvate alla I conferenza nazionale dei Gruppi Anarchici d'Azione Proletaria, tenuta a Genova - Pontedecimo nei giorni 24-25 febbraio 1951), Genova, Comitato Nazionale dei G.A.A.P., 1952; CERRITO, Gino, Il ruolo della organizzazione anarchica. L'efficientismo organizzativo, il problema della minoranza, il periodo transitorio, classismo e umanesimo, Catania, RL, 1973.

5 IISGA (Internationaal Instituut voor Sociale Geschiedenis, Amsterdam), fondo Fedeli, b. 126, Breve relazione sul movimento anarchico in Italia, s.d. [1951].
} 
quali le più importanti sono: La Federazione Toscana, con sede in Livorno, quella Romagnola, con sede in Ravenna, la Marchigiana con sede in Ancona, la Federazione Ligure, in Genova, ecc. ma vi sono poi altre numerosissime federazioni $[\ldots]$.

Il V congresso FAI (Civitavecchia, 1953) approva risoluzioni inerenti a una concezione "rivoluzionaria e educativa insieme" dell'anarchismo, rifiutando sia l'individualismo che la lotta di classe, richiamandosi allo "spirito" di Saint-Imier del 1872. Contro i GAAP «[...] corrente nefasta negatrice dell'anarchismo che sembra amalgamare la mentalità marxista». È l'atto di nascita della "FAIMovimento". ${ }^{6}$

Il 1956, anno indimenticabile, apre alle concezioni libertarie del socialismo e crea nuovi spazi per l'anarchismo. Al VI congresso (Senigallia, novembre 1957) la FAI si struttura in "gruppi di lavoro": anticlericalismo e cultura, antifascismo, movimento operaio e progresso tecnologico, «lotta contro il bolscevismo, contro le sue posizioni dittatoriali e controrivoluzionarie», mondo giovanile7. Si avverte un certo dinamismo intorno all'iniziativa dei Campeggi internazionali (che edita il bollettino «Lotta Anarchica»).

Al VII congresso FAI (Rosignano Solvay, giugno 1961) si potenziano gli sforzi finanziari per la stampa, si pone attenzione all'esperienza sindacale USI già funzionante a Carrara, si dà voce ai movimenti culturali giovanili, a quelli artistici. Si conferma l'opposizione alla politica dei blocchi, al neofascismo, al totalitarismo clericale e al comunismo autoritario ${ }^{8}$.

Uno spirito mobilitante anima i gruppi. Nel 1962 a Torino i ragazzi del "Libero Accordo" partecipano alla rivolta di piazza Statuto, al corteo spontaneo degli operai FIAT contro la UIL, agli scontri con la polizia. La tensione tocca l'apice con il rapimento di Isu Elias, vice-console spagnolo a Milano, effettuato da giovani libertari italiani per chiedere, in cambio della liberazione dell'ostaggio, che sia garantita la vita all'anarchico

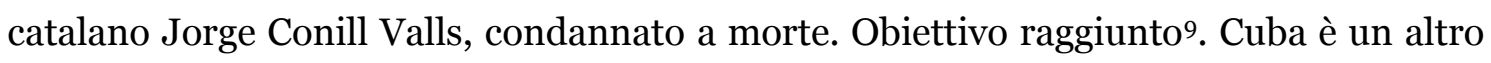

\footnotetext{
${ }^{6}$ Cfr. «Il congresso di Civitavecchia», in Volontà, n. 3/1953. I GAAP si disperderanno nella galassia dissidente rivoluzionaria e socialdemocratica.

${ }_{7}$ Cfr. Bollettino Interno della FAI, n. 1/1957 e n. 2/1958.

${ }^{8}$ Cfr. «Relazione della C.d.C.», in Bollettino Interno della FAI, n. 40-41/1961.

${ }_{9}$ Cfr. Corriere della Sera, set.-ott. 1962, pass.
} 
argomento che suscita discussioni: sulla natura dittatoriale del regime castrista, sull'ipotesi di autonomia dell'isola da ambedue i blocchi imperialistici ${ }^{10}$.

L'VIII congresso della FAI (Carrara, novembre 1965) registra la rottura tra "adunatisti" e "strutturatori”. Il nuovo patto associativo, stilato sulla falsariga del patto dell'UAI del 1920, è approvato mentre dissidenti vicini agli "antiorganizzatori” italoamericani si distaccano fondando i GIA ${ }^{11}$.

\section{Tendenze anarcosindacaliste nel movimento operaio ${ }^{12}$}

Alla rifondazione del sindacato (Patto di Roma, 8 giugno 1944), la corrente anarchica dispone di prestigiosi organizzatori, leaders storici di minatori, cavatori, metallurgici, ferrovieri, ecc., già militanti nell'USI: Attilio Sassi, Alberto Meschi, Gaetano Gervasio, Augusto Castrucci, Umberto Marzocchi e altri. Fautori del conflitto sociale dal basso e dell'autogestione, contrari al centralismo confederale, intendono cambiare i connotati del sindacato, operando nelle sue strutture ${ }^{13}$.

Scelta "entrista" non da tutti condivisa. Al sud l'Alleanza dei Gruppi Libertari dichiara la sua avversità alla CGIL, «prosecuzione dello pseudo sindacalismo totalitario e oppressivo del fascismo», prefiggendosi di promuovere sindacati alternativi. Tentativi in tal senso si erano avuti fin dal '43, nel napoletano. Comunisti dissidenti, socialisti, azionisti, sindacalisti rivoluzionari avevano promosso la ricostituzione della CGL (senza la "I" nella sigla) "rossa" e indipendente. Esperienza classista e di opposizione ad ogni politica di unità nazionale subito bloccata dal PCI.

Collaborazione, produttivismo, Nazione: questi i capisaldi del nuovo sindacato, che rinuncia a porsi come forza sovversiva. Patria e lavoro: nella CGIL unitaria si sancisce il monopolio degli apparati comunista, socialista e democristiano. Gli anarchici, esclusi dai vertici confederali - dove avrebbero voluto un loro quarto segretario generale -

\footnotetext{
${ }^{10}$ Cfr. Boletìn de Informaciòn Libertaria, Miami, aa. 1962-1963, pass.

${ }^{11}$ Tra FAI e GIA («L'Internazionale») vi saranno anche contrasti nella gestione finanziaria della Colonia "Maria Luisa Berneri", benemerita istituzione educativa.

12 Il paragrafo è un'anteprima di SACCHETTI, Giorgio, Lavoro, democrazia, autogestione. Correnti libertarie nel sindacalismo italiano (1944-1969), Roma, Aracne, in corso di pubblicazione.

${ }^{13}$ Cfr. MARABINI, Tomaso, SACCHETTI, Giorgio, ZANI, Roberto, Attilio Sassi detto Bestione. Autobiografia di un sindacalista libertario (1876-1957), Milano, Zero in condotta, 2008; GERVASIO, Gaetano, GERVASIO, Giovanna, Un operaio semplice. Storia di un sindacalista rivoluzionario anarchico (1886-1964), Milano, Zero in condotta, 2011; SACCHETTI, Giorgio, Senza frontiere. Pensiero e azione dell'anarchico Umberto Marzocchi (190o-1986), Milano, Zero in condotta, 2005.
} 
ottengono solo una rappresentanza nel Comitato direttivo (su 15 membri) con Attilio Sassi, il "capo" dei minatori italiani.

Al convegno sindacale dell'Italia liberata (1944) si discute di rappresentanza operaia. Partecipano sindacati americani e Fédération Syndicale Mondiale (FSM). Sassi vi interviene a reclamare autonomia, per un libero sindacalismo di classe. Concetto ribadito all'assise nazionale di Napoli (1945) dove chiede, come vincolo per i nuovi contratti, l'approvazione preventiva dei lavoratori. Aderisce alla CGIL anche il ricostituito Sindacato Ferrovieri Italiani (SFI). Castrucci, storico leader dei macchinisti (fondatore dell' «In Marcia!») è eletto segretario nazionale di categoria e poi segretario generale onorario SFI, promotore della Federazione Apartitica (FASFI), con l'intento di affermare l'indipendenza del sindacato ${ }^{14}$.

Con gli Alleati in procinto di sfondare la Linea gotica e gli scioperi insurrezionali in atto, il Comitato di Liberazione Nazionale Alta Italia (CLNAI) converte i CLN aziendali in "consigli di gestione". La FCL - «riconosciuta la necessità contingente dell'unità sindacale» - partecipa alle strutture democratiche di fabbrica; le sue liste ottengono il $4 \%$ (oltre 17.000 voti) a Milano dove gli anarchici hanno un ruolo importante nella FIOM; a Genova il 3,6\% fra i metalmeccanici e il 2,5\% fra i portuali.

Si delinea la «Ideologia della Ricostruzione», progetto che accomuna imprenditori, sindacato e partiti, presupposto per una politica di contenimento salariale e alta produttività. Per gli anarchici una vera rinuncia alla lotta. La FAI promuove un Comitato Nazionale di Difesa Sindacale (CNDS).

Lo "spirito del '45" è finito. Nel direttivo eletto al congresso di Firenze del 1947 risulta un solo anarchico. Uno su 75 membri. Nel SFI la componente libertaria conta 3669 iscritti, 4711 nella FIOM, 1327 nella FILEA edili, 792 nella FNDS statali, 530 nella Federterra, per un complessivo $1 \%$ in tutta la CGIL. Gli anarchici come corrente, a parte le posizioni individuali di alcuni dirigenti, perdono di importanza nella CGIL.

La "confederalità", dimensione politica e di controllo preventivo sulle rivendicazioni, è la bestia nera dei libertari. Su diritto di sciopero, sindacalismo federale e autodeterminazione delle categorie si consuma l'estremo duello. Gli anarchici sostengono la validità erga omnes dei risultati conseguiti sul territorio nazionale ed iter contrattuali partecipati. Sottolineano il dovere inderogabile della rappresentanza di classe. Coordinato da Gervasio, il CNDS agisce come corrente dei «Gruppi di Difesa Sindacale rivoluzionaria per una CGIL classista, libera ed indipendente dai partiti e

${ }^{14}$ La corrente apartitista (sindacalisti rivoluzionari e anarchici) ottiene il $2 \%$ al congresso SFI del 1948, sarà sciolta nel 1949. 
dallo Stato». Mantiene rapporti con l'AIT che svolge un ruolo di raccordo mondiale fra le organizzazioni operaie libertarie. E, tuttavia, continua «a far parte della CGIL che ufficialmente aderisce alla FSM». Nel 1947 si consuma la rottura nella roccaforte libertaria di Carrara. Meschi, per evitare lo scontro con i socialcomunisti si dimette da segretario della Camera del lavoro⒌ Per Sassi l'unica via rimane l'unità “dal basso", attraverso le occupazioni delle terre e gli scioperi spontanei.

La guerra fredda, intanto, si insinua nella società europea.

Fra CGIL e progetto USI l'incertezza rimane, mentre si fa strada una terza opzione: «La morte del sindacalismo in quanto possibilità rivoluzionaria è un fatto che diventa sempre più chiaro nella coscienza di tutti i militanti $[\ldots]{ }^{16}$.

La CGIL è accusata dai suoi dirigenti libertari di essere «buona solamente per scopi politici»: lo statuto del 1945 ha partorito burocrati accentratori e autoritari.

Sconfitto il Fronte alle elezioni del 18 aprile, termina il ciclo straordinario della CGIL unitaria. All'attentato a Togliatti seguono tentativi insurrezionali, con arresti di partigiani e sindacalisti. Lo sciopero generale, sconfessato dalla DC, porta alla scissione effettiva. Banco di prova le categorie. Al congresso dei minatori la lista «unitaria» (socialcomunisti, PSLI, repubblicani, anarchici) mette in minoranza i democristiani. Fra i ferrovieri, categoria strategica, Castrucci suggella con un «Addio compagni...» la partecipazione alla dirigenza SFI-CGIL.

Il sindacalismo europeo si orienta verso la industrial democracy. Qualcuno vorrebbe ora imbarcare gli anarchici, classisti ma pur sempre "anticomunisti”. È il caso dei fuoriusciti PSLI e repubblicani che fondano la Federazione Italiana del Lavoro (FIL). Sassi e Meschi smentiscono ribadendo la funzione unitaria dei libertari nella CGIL.

Il gruppo «Milano-1» convoca un convegno per costituire «una organizzazione dei lavoratori di tipo spagnolo sulle linee dell'AIT». Si riaffaccia l'ipotesi USI. Il dato di analisi è che l'esperienza fascista ha esasperato il carattere statale dei sindacati e l'influenza comunista nella CGIL ha confermato tale fisionomia ${ }^{17}$.

Il congresso confederale di Genova (1949) propone un Piano del Lavoro d'impronta keynesiana riformista, con obiettivi non lontani da quelli del Piano Marshall. Sassi

${ }_{15}$ Cfr. ROLLAND, Hugo, Il sindacalismo anarchico di Alberto Meschi, Firenze, La Nuova Italia, 1972, pp. 98-99.

16 BERNERI, Giovanna, TURRONI, Pio, ZACCARIA, Cesare, «Residui del sindacalismo», in Volontà, n. 4-5, 15 nov. 1948.

${ }_{17}$ Cfr. L'Anarchismo e i lavoratori. Un convegno di studi sui rapporti fra movimento anarchico e movimento dei lavoratori, Milano, edizioni G.M. 1, 1950. 
interviene per dissentire da ogni filosofia collaborativa. Ridisegnata la geografia delle correnti: Unità sindacale (PCI); Unità e democrazia sindacale (PSI); Socialisti democratici; Cristiani unitari; indipendenti; movimento unitario Mazziniano; corrente Sindacalista. In quest'ultima sono inseriti, d'ufficio, gli anarchici. Nasce il "vero" sindacato dell'Italia repubblicana, fondato su un assetto sistemico tripolare. Per gli anarchici: o i CNDS, minoranza inerme nella CGIL; o l'USI, formalmente ricostituita.

Sassi, Gervasio, Marzocchi restano i referenti per l'area libertaria nel sindacato socialcomunista. L'esperienza, ora senza il sostegno della FAI, è ritenuta ancora valida. Gli obiettivi (riduzione dell'orario di lavoro, autonomia dai partiti, contratti previo consenso della base) tutti da realizzare. Ripartire da luoghi di lavoro e categorie: conflitto e contratto. A Napoli, III congresso CGIL (1953), interviene la corrente anarcosindacalista. «[...] Si doveva restare estranei alla competizione politica [...]»: Sassi dissente dal direttivo che, al contrario, rivendica il contributo dato per la battaglia delle sinistre contro la «legge truffa» (legge elettorale maggioritaria) ${ }^{18}$.

A Roma, IV Congresso CGIL (1956), Difesa Sindacale presenta un proprio documento: contro l'indirizzo produttivistico confederale e per le 36 ore, per le libertà sindacali, la democrazia interna e il rispetto dello statuto...19 Esito risibile. Prevale lo "stile freddo dei funzionari", a parte l'intervento dell'ottantenne Sassi. Che denuncia gli errori, nell'accettare la scala mobile ("miseria stabile") e il regolamento per le commissioni interne, nell'impostare le lotte «con un minimo di sacrificio, con la speranza vana di un massimo risultato».

La temperie internazionale ed il "nuovo corso" per la sinistra sindacale trovano gli anarchici (non solo loro) impreparati.

La CGIL possiede i migliori quadri sindacali del movimento operaio. Se fossimo stati forti nel 1956, al momento dei fatti d'Ungheria avremmo potuto tentare una scissione a favore dell'autonomia o dell'USI $[\ldots]^{20}$

${ }^{18}$ Cfr. Notiziario CGIL, III, 1949; Notiziario CGIL, VII, 1953; Notiziario CGIL, VIII, 1954. Per Difesa Sindacale 4.188 voti all'elezione del comitato direttivo nel ' 49 , meno dell'1\%.

${ }_{19}$ Cfr. Dichiarazione della corrente anarchica di Difesa Sindacale al IV Congresso della CGIL, Genova, Tip. Coop. Poligrafici, 1955.

${ }^{20}$ MARZOCCHI, Umberto, «Panorama syndical de l'Italie: Gli anarchici ed il movimento operaio dopo la caduta del fascismo», in Bulletin CILO, 7/1959. 


\section{L'Internazionale anarchica ${ }^{21}$}

Il movimento anarchico ha sempre perseguito, con fortune alterne, una sua dimensione organizzativa internazionale. Nel XIX secolo i seguaci di Bakunin si coalizzano, separandosi da Marx e Mazzini, nell'Alleanza della Democrazia Socialista prima, nella corrente autonomista federalista in seno alla Prima Internazionale poi. Esperienze basilari queste, fino al congresso di Saint Imier (1872).

Il Novecento è marcato da eventi rivoluzionari che graveranno sull'eredità anche organizzativa del milieu libertario. Con Errico Malatesta nel 1907 ad Amsterdam, Ugo Fedeli nel 1921 a Berlino e ancora nel 1949 a Parigi, poi nel 1958 a Londra con Umberto Marzocchi (stando ai protagonisti italiani) si consumano generosi tentativi. Ma l'Internazionale di Federazioni Anarchiche (IFA) si concretizzerà solo con il congresso di fondazione (Carrara, 1968) ${ }^{22}$.

Tutto inizia in Francia, dove è presente una nutrita colonia di perseguitati dell'Est europeo, di antifranchisti iberici, israeliti, gruppi cosmopoliti, esperantisti... Ai "soci fondatori" si aggiungeranno le aree latino-americana e scandinava (a tradizione anarcosindacalista), quella anglofona e tedesca, italo-americana, di cultura ebraica, asiatica (giapponese, cinese di Hong Kong, coreana...), australiana, ecc.

Primi due appuntamenti: una Conferenza pan-americana a Montevideo nel 1957, il congresso di Londra l'anno dopo. I risultati del consesso uruguagio sono importanti: rapporti permanenti attraverso una segreteria tecnica centralizzata; biblioteche libertarie, archivi storici e case editrici; partecipazione coordinata all'attività sindacale purché indipendente; soccorso ai perseguitati. A Londra sono stabilite le modalità di funzionamento della "Internazionale Anarchica", madre dell'IFA.

A Carrara nel 1968 (presenti 34 delegazioni nazionali da quattro continenti) i vecchi militanti come Failla, Mantovani, Marzocchi - sostenuti dai rappresentanti delle altre federazioni - si confrontano con i giovani, con Daniel Cohn-Bendit, portavoce del gruppo francese “22 Marzo”. Accusato di cedere a suggestioni situazioniste marxiste, il

${ }^{21}$ Per questo paragrafo: SACCHETTI, Giorgio, L'Internazionale anarchica: mappe e percorsi nel secondo Novecento, relazione (inedita) al convegno di studi storici "Alla prova del '68. L’anarchismo internazionale al congresso di Carrara», Imola, 8 novembre 2008.

${ }^{22}$ Cfr. CRIFA (Commission de relations de l'Internationale des fédérations anarchistes), Recueil des résolutions adoptées par les Congrès anarchistes internationaux: Londres (25juillet-1 aout 1958), Carrare (31 aout-5 septembre 1968), Paris (1-4 aout 1971), Carrara, La Tipografia Il Seme, 1977. 
leader studentesco ribadisce le sue concezioni di una «azione basata sulla spontaneità» per «abbandonare il falso dilemma: anarchici o marxisti, per porre il dilemma: rivoluzionari o no». Il contrasto rasenta la rissa fino a che Cohn-Bendit non abbandona la scena. Del suo gauchisme rimarrà poco nella prassi anarchica. Le risoluzioni congressuali negheranno qualsiasi valenza positiva al "socialismo" realizzato, dittatura castrista e modello cinese compresi.

Volendo tracciare una mappa geopolitica dell'anarchismo internazionale è possibile individuare tre grandi famiglie.

Della prima fanno parte entità «fondate sul principio dell'associazione permanente e che, nella determinazione del programma tattico, attribuiscono un valore essenziale ai congressi». Fra queste il Movimento Libertario spagnolo (MLE) che comprende: FA Iberica, CNT, Solidaridad Internaciònal Antifascista (SIA), Mujeres Libres e FIJL. Poi ci sono: la FACB bulgara (cui aderisce la UAB in esilio), la FLA argentina, le federazioni di Messico, Perù, Uruguay, Corea, il Movimento Libertario Cubano in esilio.

Del secondo gruppo fanno parte «organizzazioni di tendenza sia pure permanenti, che talora si identificano con il movimento generale», in Italia FAI, FAGI (Federazione Anarchica Giovanile Italiana) e GIA, la FA francese, la "Federazione del Nord” AFN di Svezia, Norvegia, Finlandia e Danimarca, il Movimento Anarchico di lingua e cultura yiddish, i gruppi israeliani "Problemot", ecc.

Nell'ultimo raggruppamento vi sono quelle compagini che «non rispondono a organizzazioni permanenti (per ragioni talvolta giustificate dalla situazione obbiettiva dei rispettivi paesi) e considerano i congressi semplici incontri utili per scambi di opinioni o necessari per occasionali accordi». Come ad esempio: i movimenti anarchici del Belgio (MAB), della Gran Bretagna (AMGB), della Cina (CAM), le Federazioni Socialiste Libertarie dell'Olanda (FVS), della Svizzera (FSLS), le Federazioni Anarchiche della Germania (DAF), della Grecia (HAKE), del Giappone (JAF), alcune federazioni sudamericane, vari gruppi informali giovanili del nord Europa e dei paesi dell'Est, ecc. Inoltre, al di fuori di queste tre "famiglie", vi sono centinaia di formazioni nel mondo inquadrabili, in linea di massima: nell'anarchismo nonviolento (pacifisti indiani di Vinoba); nell'anarcosindacalismo; nella sperimentazione comunitaria (Comunidad del Sur in Uruguay); nelle istituzioni scolastiche libertarie; nelle fondazioni culturali, centri studi e archivi storici come il CIRA di Losanna, l'Arbatarrorelsens Arckiv di Stoccolma, l'IISG di Amsterdam; nei gruppi intellettuali che gravitano intorno alle riviste («De Vrije» in Olanda, «Noir et Rouge» in Francia...). 
Cinque le posizioni tattiche nell'anarchismo internazionale: 1) "possibilisti" moderati, vicini ai sindacati CNT in Spagna e SAC in Svezia, Gruppi yiddish, che perseguono fronti unici di opposizione senza i marxisti; 2) "educazionisti” ispirati all'umanitarismo libertario, spesso anti-organizzatori; 3) "classisti" seguaci della rivoluzione immediata globale, ispirati da tesi guerrigliere e "radicalismo fuochista" (Federaciòn Anarquica Peruana, l'omologa Uruguagia e gruppi venezuelani); 4) neorivoluzionari europei (FIJL, Black Cross, ORA francese), risorsa per il movimento ma a rischio avventurismo; 5) anarchici "tradizionali” ispirati all'organizzazione specifica con vecchi militanti e nuovissimi adepti, tra spontaneismo e classiche ricette del $\operatorname{gradualismo}^{23}$.

\subsection{Sessantotto libertario e "strategia della tensione"}

Gli anarchici prestano attenzione ai fenomeni sociali e culturali in atto, attingono al pensiero "terzomondista", vera genesi del Sessantotto. Si battono contro la fame in India e il razzismo in America. Per l'Africa la spinta anticolonialista è la vera speranza. Sulle lotte per i diritti civili le fonti spaziano, da Martin Luther King e Betrand Russel alla New Left. Sul socialismo reale si constata come, un decennio dopo il 1956, i partiti comunisti portino integro il fardello dello stalinismo. E come l'URSS e la Cina di Mao siano universi concentrazionari, che perseguitano dissidenti, artisti, scrittori e operai. Sul Vietnam martoriato dall'escalation dei bombardamenti si sostiene che «gli americani non riusciranno mai a vincere» quella sporca guerra neocolonialista.

La beat generation, i Provos d'Olanda diffondono pratiche libertarie anticonformiste: contro patria, chiesa, famiglia e partito. Per il pacifismo, la nonviolenza, la fratellanza universale, la libertà di pensiero e l'amore libero. C'è sintonia fra movimenti della contestazione e anarchici ${ }^{24}$.

A Parigi (1966) si svolge un incontro europeo dei giovani anarchici cui partecipano inglesi, belgi, spagnoli, francesi, olandesi, svedesi e italiani. Di quest'ultima delegazione fanno parte FAGI e Gruppi Giovanili Anarchici Federati (GGAF) di varie località. All'ordine del giorno: questione giovanile, Provos, mobilitazione contro la bomba

${ }^{23}$ Cfr. CERRITO, Gino, Il movimento anarchico internazionale nella sua struttura attuale. Lineamenti storici e bibliografia essenziale, in Anarchici $e$ anarchia nel mondo contemporaneo. Atti del Convegno promosso dalla Fondazione Luigi Einaudi (Torino, 5, 6 e 7 dicembre 1969), Torino, Fondazione L. Einaudi, 1971, pp. 127-207.

${ }_{24}$ Cfr. SCHIRONE, Franco, La gioventù anarchica negli anni delle contestazioni 1965-1969, Milano, Zero in condotta, 2006. 
atomica, antifranchismo, antielettoralismo, sindacalismo, organizzazione interna, campeggi internazionali.

Dopo Parigi: Milano, dove - nel dicembre 1966 - si tiene la Conferenza Europea della Gioventù Anarchica. La "tre giorni" si conclude al consolato spagnolo dove si espone una garrota in legno e si reclama libertà per gli antifascisti iberici. Un corteo è sciolto dalla polizia mentre effettua un girotondo all'albero di Natale in piazza Duomo.

Milano e Roma sono laboratori culturali giovanili, luoghi d'intrecci fra FAGI e “capelloni”. Nel capoluogo lombardo (già epicentro del caso «La Zanzara») escono i primi numeri di «Mondo Beat» e «Provo», ciclostilati in sedi anarchiche. Nella capitale i gruppi "Provos Roma 1" e FAGI “Alba Nuova” promuovono azioni solidali antifranchiste.

Siamo agli esordi tumultuosi di un "neo-anarchismo" di forte impatto. A questo periodo risale la strutturazione nazionale dei GAF, approdo della precedente esperienza di "Materialismo e Libertà". La FAGI, sigla ormai conosciuta negli ambienti studenteschi, promuove marce della pace, scioperi della fame, cortei antifascisti; collabora con Unione Goliardica Italiana, PSIUP e Partito Radicale.

A Genova (1967) si realizza un meeting fra Provos e anarchici. Il discorso prosegue a Carrara con il «primo convegno italiano della gioventù protestataria». Beatnik, Provos, "cavalieri del nulla", aderenti alla FAGI discutono di pacifismo e di comuni percorsi libertari, socializzano esperienze "on the road". Il rapporto fra "neo-anarchici" e anarchismo otto/novecentesco si consolida ${ }^{25}$.

Il IX congresso della FAI (Ancona, novembre 1967), il primo dopo la rottura con i GIA, vede un'inconsueta promiscuità generazionale.

Nelle università e nella società riaffiorano antiche istanze libertarie, luxemburghiane, anarcosindacaliste, consiliariste. L'USI rivive a Piombino, tra i cavatori di Carrara, a Genova Sestri con i portuali, a Milano Bovisa dove si stabilisce il collegamento con i Comitati di Base (CUB) dei tranvieri e alla Pirelli.

C'è una "dimensione libertaria del Sessantotto". Maggio francese e Primavera di Praga ne costituiscono la cornice europea. La sfida agli autoritarismi e, quindi, anche al "totalitarismo comunista" trova rispondenza nella FAI. C'è sintonia fra reduci anarchici della Spagna del 1936 e quella parte della gioventù ribelle che marca una ragguardevole distanza dall'ortodossia marxista. Contro il dispotismo "di sinistra" si consolida la

25 Cfr. Rencontre européenne des Jeunes Anarchistes, Paris, 13/4/1966, in Archivio BFS, Pisa; e Bollettino Europeo della Gioventù Anarchica, n. 3/1967. 
battaglia dei libertari, al loro fianco una sparuta pattuglia di dissidenti (da Lelio Basso ai giovani Marco Boato e Adriano Sofri).

Siamo alle avvisaglie dell'autunno caldo. Nel 1969 si verificano in Italia 145 attentati. Per le bombe del 25 aprile alla Fiera di Milano sono accusati gli anarchici; e forte sarà la mobilitazione per reclamarne l'innocenza.

Il 12 dicembre 1969 a Milano una bomba esplode alla Banca Nazionale dell'Agricoltura in piazza Fontana. Il massacro, 16 morti e 87 feriti, scuote il paese. Il giudice Amati, il questore Guida, il commissario Calabresi individuano subito la "pista anarchica”, ipotesi supportata dall'Ufficio affari riservati di F. Umberto d'Amato. È arrestato il "ballerino anarchico" Pietro Valpreda (farà tre anni di carcere in attesa di processo) cui viene contestato, in base alla testimonianza di un tassista, di essere l'autore del massacro. Illegalmente trattenuto in questura Giuseppe Pinelli, ferroviere del circolo Ponte della Ghisolfa, ne uscirà dopo tre giorni "volando" dal quarto piano. Il mattino seguente i giornali titolano: "suicidio". Ma già si delineano verità differenti da quelle ufficiali. Lotta Continua, anarchici ed extra-parlamentari accusano fascisti e servizi segreti della strage. Anche l'opinione pubblica democratica si schiera. La parola d'ordine, per anni, sarà: «Valpreda è innocente, la strage è di Stato, Pinelli è stato assassinato» ${ }^{26}$.

Perché proprio gli anarchici? La tesi più diffusa, all'epoca, fu quella così detta dell'“anello debole". Trattandosi cioè di movimento non strutturato - si argomentava ne deriva l'esposizione a provocazioni. Dietro la strage di Milano c'è una parte dell'apparato statale, "per nulla deviata" e impegnata sul piano internazionale e sociale a contrastare il "comunismo" incombente. La strategia della tensione si rivela un progetto "destabilizzante per fini di stabilizzazione”. Piazza Fontana dà la stura ad una stagione stragista ("guerra a bassa intensità") caratterizzata dal connubio eversivo civile/militare fra neofascisti e gruppi oltranzisti filo-atlantici ${ }^{27}$.

Al X Congresso FAI (Carrara, aprile 1971) sono presenti 150 delegati, fra cui molti giovani. Fra le decisioni: l'affidamento della C.d.C. al gruppo "Durruti” di Firenze. Nel dopo-congresso restano questioni aperte: sulle concezioni del programma malatestiano e le risposte valide in esso ancora contenute; sul rifiuto dei metodi autoritari delle avanguardie; sul rifiuto della violenza come sistema di negazione-costruzione

\footnotetext{
${ }^{26}$ Cfr. LANZA, Luciano, Bombe e segreti. Piazza Fontana 1969, Milano, Eleuthera, 1997.

27 Cfr. CUCCHIARELLI, Paolo, GIANNULI, Aldo, Lo Stato parallelo. L'Italia 'oscura' nei documenti e nelle relazioni della Commissione Stragi, Roma, Gamberetti, 1997.
} 
rivoluzionaria e della sua accettazione solo come extrema ratio, "nei limiti dello stretto necessario".

Su quest'ultima insidia teorica, però, le posizioni non sono sempre univoche. La C.d.C. dichiara di appoggiare la banda Baader-Meinhof, avallandone le posizioni e ritenendole compatibili da un punto di vista libertario. È l’ideologia dei gruppi "22 Marzo", della rivoluzione come atto di vendetta e di rabbia. I nodi vengono al pettine in occasione di una manifestazione indetta a Milano per l'11 marzo 1972 da Avanguardia Operaia, Lotta Continua, Potere Operaio, gruppo Gramsci e IV Internazionale, nell'ambito della campagna Valpreda. «Umanità Nova», GAF e Organizzazione Anarchica Milanese rifiutano di aderire all'iniziativa ritenendola avventuristica. Gli scontri con la polizia che poi in effetti si verificano, e le conseguenze per chi è stato mandato allo sbaraglio, suscitano recriminazioni. La C.d.C. fiorentina denuncia l'opportunismo dei rinunciatari e rivendica la coerenza anarchica del fatto insurrezionale e dello scontro di piazza. Le posizioni guerrigliere del "Durruti", appoggiate dal gruppo siciliano "La Sinistra Libertaria", non hanno seguito nella FAI (che approva un documento di rifiuto del terrorismo). Epilogo: dimissioni del gruppo di Firenze e nomina di una nuova C.d.C.

Per le elezioni politiche del 1972 un convegno generale del movimento ribadisce, dopo la candidatura-protesta di Valpreda promossa da «Il Manifesto», la posizione astensionista. Contro la montatura statale poliziesca non servirà la guerriglia, ma nemmeno il voto ${ }^{28}$.

Il 1972-1973 è segnato da nuovi fatti cruenti. A Pisa muore a vent'anni Franco Serantini massacrato dalla polizia mentre manifesta contro un comizio del MSI, abbandonato in carcere senza cure. A Milano è ucciso il commissario Calabresi (la responsabilità dell'omicidio sarà addossata ai vertici di Lotta Continua).

Poi la vicenda di Giovanni Marini - l'anarchico salernitano responsabile dell'uccisione del neofascista Carlo Falvella durante una colluttazione - su cui «Umanità Nova» espone una tesi inquietante.

[...] c'è anche un motivo ben preciso per cui Marini fu aggredito la notte del sette luglio; un motivo che trova legami non lontani con gli attentati di Gioia Tauro e del dicembre '69. Il 15 dicembre 1970, infatti, sull'Autostrada del Sole, circa all'altezza della villa di Junio Valerio Borghese avveniva un incidente in circostanze misteriose. Un camion, guidato da un fascista salernitano, si scontrava con un'auto

${ }^{28}$ Cfr. Bollettino Interno della FAI, 1972, pass. 
dentro cui viaggiavano cinque anarchici, uccidendoli. Essi erano diretti a Roma per consegnare ad un avvocato i risultati delle indagini da loro svolte sulle attività dei fascisti in Calabria ed in particolare le prove che il deragliamento della Freccia del Sud (6 morti) era stato provocato dai fascisti. Tre erano testi del processo Valpreda: Angelo Casile, Gianni Aricò e Annalise Borth. Tutti e cinque rimasero uccisi nello scontro; dell'inchiesta non si seppe più nulla. Su questi fatti Giovanni Marini stava indagando $[\ldots]^{29}$

Il 17 maggio 1973 Gianfranco Bertoli, sedicente anarchico, lancia una bomba davanti alla questura di Milano causando quattro morti e 45 feriti fra i passanti. L'intenzione sarebbe stata quella di attentare alla vita del ministro dell'interno Rumor, lì presente fino a poco prima per commemorare Calabresi ${ }^{30}$.

L'anarchismo dei primi anni settanta, mentre mantiene una peculiare presenza attiva nei movimenti, rinvigorendo le tradizionali campagne antifasciste in Italia ed in ambito internazionale (Spagna, Grecia, Cile...), opera scelte organizzative che avranno impatto di lunga durata.

Da una parte la FAI conferma il suo rifiuto nei confronti di ogni accentuato classismo e dei principi di responsabilità collettiva e dell'unità ideologica, ancora prospettati dai cosiddetti "neo piattaformisti" (una trentina di gruppi, riuniti a Bologna nell'agosto 1973, nel I convegno nazionale dei lavoratori anarchici) ${ }^{31}$. Dall'altra si ridefiniscono le modalità di partecipazione degli anarchici al movimento operaio: ricostituire sezioni territoriali dell'USI oppure, in subordine, promuovere "Comitati di azione diretta di opposizione al riformismo sindacale" ${ }^{2}$.

29 «Umanità Nova», 16 giu. 1973, Perché Marini fu aggredito. Anche uno dei destinatari del dossier (Aldo Rossi, redattore di «Umanità Nova») rimarrà vittima di lì a poco di un incidente stradale su cui restano dubbi.

$3^{30}$ Cfr. Bollettino Interno della FAI, n. 14, 31 mag., 1973. Per l'attentato sono condannati, oltre l'autore materiale, fascisti di "Ordine Nuovo".

${ }^{31}$ Cfr. Bollettino del I CNLA, 1973, ciclostilato, ora in DADÀ, Adriano, L'anarchismo in Italia: fra movimento e partito. Storia e documenti dell'anarchismo italiano, Milano, Teti, 1984, pp. 381-382.

${ }^{2}$ Cfr. Bollettino Interno della FAI, n. 14, 31 mag., 1973, pass.; [FINZI, Paolo], «Anarchici oggi», in A rivista anarchica, n. 26, gen.-feb., 1974; ROSSI, Italino, "Analisi per una storia della Federazione Anarchica Italiana», in Umanità Nova, 27 apr./1 mag. 1986; SACCHETTI, Giorgio, Senza frontiere, cit., pp. 217-238. 


\section{* L'autore}

È dottore di ricerca in Storia del movimento sindacale, cultore della materia presso la cattedra di Storia dei partititi e dei movimenti politici all’Università di Trieste. Già direttore della «Rivista Storica dell'Anarchismo»; membro dei comitati scientifici Archivio fam. Berneri - A. Chessa (Reggio Emilia) e «Germinal - Revista de Estudios Libertarios» (Madrid); redattore del Dizionario Biografico degli anarchici italiani (BFS, 2003-2004). Si occupa di labour history e di culture libertarie del Novecento. L'ultimo volume da lui pubblicato è Sovversivi e squadristi. 1921: alle origini della guerra civile in provincia di Arezzo, Roma, Aracne, 2010.

URL: < http://studistorici.com/progett/autori/\#Sacchetti >

\section{Per citare questo articolo:}

SACCHETTI, Giorgio, «Eretici e libertari. II movimento anarchico in Italia (1945-1973)», Diacronie. Studi di Storia Contemporanea: Quando la classe operaia andava in paradiso, 13/2/2012, URL:< http://www.studistorici.com/2012/02/13/sacchetti_numero_9/ >

Diacronie Studi di Storia Contemporanea 3 www.diacronie.it

Risorsa digitale indipendente a carattere storiografico. Uscita trimestrale. redazione.diacronie@hotmail.it

Comitato di redazione: Marco Abram - Giampaolo Amodei - Jacopo Bassi - Luca Bufarale - Alessandro Cattunar - Alice De Rensis Barbara Galimberti - Deborah Paci - Fausto Pietrancosta - Martina Sanna - Matteo Tomasoni - Luca Zuccolo

Diritti: gli articoli di Diacronie. Studi di Storia Contemporanea sono pubblicati sotto licenza Creative Commons 2.5 Possono essere riprodotti a patto di non modificarne i contenuti e di non usarli per fini commerciali. La citazione di estratti è comunque sempre autorizzata, nei limiti previsti dalla legge. 